\title{
Learning rule sets from survival data
}

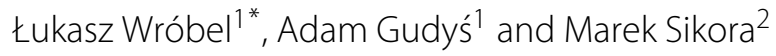

\begin{abstract}
Background: Survival analysis is an important element of reasoning from data. Applied in a number of fields, it has become particularly useful in medicine to estimate the survival rate of patients on the basis of their condition, examination results, and undergoing treatment. The recent developments in the next generation sequencing open new opportunities in survival study as they allow vast amount of genome-, transcriptome-, and proteome-related features to be investigated. These include single nucleotide and structural variants, expressions of genes and microRNAs, DNA methylation, and many others.

Results: We present LR-Rules, a new algorithm for rule induction from survival data. It works according to the separate-and-conquer heuristics with a use of log-rank test for establishing rule body. Extensive experiments show LR-Rules to generate models of superior accuracy and comprehensibility. The detailed analysis of rules rendered by the presented algorithm on four medical datasets concerning leukemia as well as breast, lung, and thyroid cancers, reveals the ability to discover true relations between attributes and patients' survival rate. Two of the case studies incorporate features obtained with a use of high throughput technologies showing the usability of the algorithm in the analysis of bioinformatics data.

Conclusions: LR-Rules is a viable alternative to existing approaches to survival analysis, particularly when the interpretability of a resulting model is crucial. Presented algorithm may be especially useful when applied on the genomic and proteomic data as it may contribute to the better understanding of the background of diseases and support their treatments.
\end{abstract}

Keywords: Survival analysis, Separate-and-conquer, Rule induction, Log-rank test, High throughput sequencing, Cancer

\section{Background}

Modeling the impact of covariates on survival time is an important task of survival analysis. The most popular approaches to this problem are parametric [1] and semi-parametric statistical techniques like Cox proportional hazards regression [2] and its extensions. However, restrictive assumptions made by these strategies and difficulty in representing nonlinear interactions between covariates are one of the motivations for developing new methods based on machine learning techniques. The application of machine learning to survival analysis usually allows overcoming the limitations of statistical methods. In this paper we investigate a nonparametric rule-based approach to modeling survival data.

\footnotetext{
*Correspondence: lukasz.wrobel@polsl.pl

${ }^{1}$ Institute of Informatics, Silesian Univ. of Technology, Akademicka 16, 44-100 Gliwice, Poland

Full list of author information is available at the end of the article
}

Rule induction is one of the oldest and most frequently used methods of machine learning. Although numerous successful applications in a wide range of predictive and descriptive data mining tasks, there is still a little research on rule learning in survival analysis. Naturally, in the case of absence of censored observations the standard rulebased regression [3-5] techniques can be applied. However, as the overwhelming majority of survival datasets contains censored instances, the methods able to handle censored data are of great value. In this paper we investigate rule induction algorithm in combination with the log-rank statistical test [6]. This nonparametric test is used to compare the survival distributions of two samples and is appropriate for censored data analysis. In our study the test is used to establish the key factors affecting overall survival time of observations covered by the rules being induced. As the basis of rule induction method we selected a separate-and-conquer (known also as covering) 
strategy $[7,8]$ which is one of the most common heuristics for induction of classification rules.

\section{Related work}

Methods of survival analysis are mainly used in medical studies. Although rule-based algorithms are often applied in medical research, there is a relatively small number of papers concerning the application of rule induction to survival analysis.

Pattaraintakorn and Cercone [9] describe the rough setbased intelligent system for survival analysis. The model construction relies on a so-called minimal decision rule induction algorithm for identification of the main factors affecting survival time of patients. The survival time is considered as a discrete variable with predefined values (e.g. survival time between 56 and 73 months) dividing an entire dataset into separate decision classes.

The rough set-based approach to survival analysis is also the subject of the Bazan et al.s work [10]. For each observation in the analyzed dataset, a prognostic index (PI) based on the Cox's proportional hazard model is calculated. A range of PI values is divided into three intervals, thereby creating separate groups differing in the survival rate, and the rules are induced for resulting classes.

Sikora et al. [11] applied rule induction algorithm to the analysis of patients after bone marrow transplantation. The set of patients is divided into three groups: the patients for whom at least 5 years have passed since the transplantation (the class alive), the patients who died within 5 years after transplantation (the class dead), and the patients who are still alive but their survival time is less than 5 years (the class alive-5). Rules are generated for dataset containing alive and dead classes, whereas the alive- 5 is used for the post-processing of obtained rules.

Kronek and Reddy [12] proposed the extension of Logical Analysis of Data (LAD) [13, 14] for survival analysis. The LAD algorithm is a combinatorial approach to rule induction. It was originally developed for the analysis of data containing binary attributes, therefore the data preprocessing by discretization and binarization methods is usually required.

Liu et al. [15] adapted patient rule induction method to the analysis of survival data. The method uses so-called bump hunting which creates rules by searching regions in covariates space with a high average value of the target variable. To deal with censoring, the authors use deviance residuals as the outcome variable. The idea of residualbased approach to censored outcome is derived from survival trees $[16,17]$.

Wróbel [18] proposed to use a survival tree for induction of an ordered set of rules (decision list) from survival data. The core idea is to learn a survival tree, extract the best rule from it, and remove observations which are covered by the rule. The procedure is recursively repeated for remaining observations. This idea follows the approach used by the PART [19] and M5Rules [3] algorithms for learning classification and regression rules, respectively.

Wróbel and Sikora [20] investigated a separate-andconquer method of rule induction in combination with a weighting scheme for handling censored observations. Each observation is assigned an appropriate weight to a positive or negative class. The positive class represents observations with high risk of event occurrence, whereas negative class includes potentially event-free ones. If observation have experienced an event, then it belongs to the positive class with weight equal to 1 . Censored instances are assigned to both classes, but with different weights. The observations censored earlier receive higher weight for the positive class than the observations censored later. In the experimental study the authors pay special attention to rule quality measures [21-23] which are one of the key elements of rule induction algorithms.

It should be noted that the aforementioned studies primarily concern the application of rule-based survival analysis to usually one, particular dataset. Pattaraintakorn and Cercone [9] mainly focused on geriatric data of Canadian patients, Bazan et al. [10] analyzed data of patients with various kinds of the head and neck cancer cases, Sikora et al. [11] studied the effects of bone marrow transplantation, Liu et al. [15] performed an analysis of kidney cancer tissue microarray data. Kronek and Reddy [12] proposed a more general approach, however they verified the algorithm for only two real-life datasets. The exceptions are our previous work $[18,20]$ where survival tree-based and weighted separate-and-conquer algorithm for rule induction were tested on over a dozen various survival datasets.

There are also machine learning methods dedicated to censored data analysis and not associated with the rule induction. These are trees [16, 24-26], neural networks [27-29], bayesian networks [30, 31], support vector machines (SVM) [32], and ensemble approaches [33-35]. Among all aforementioned methods, the most widely used are tree-based techniques called survival trees.

Survival trees are an adaptation of classification and regression trees [36] to the problem of survival. In comparison to rule-based techniques, tree-based methods received much more attention in survival analysis [26]. On the other hand, a tree can be easily represented in the form of a set of rules where each path from the root to the leaf of the tree corresponds to one rule, thus it can be considered as a special case of the rule-based model. The key idea of the application of tree-based techniques to survival data lies in the splitting criterion [37]. The most popular approaches are residual-based ones $[16,17]$ as well as methods employing log-rank statistics $[25,38]$ for the maximization of the difference between survival distributions of child nodes. While searching for optimal splitting 
point with the use of the log-rank criterion, resampling methods are used too [39]. The extension of the decision trees idea are decision tree ensembles which includes, for example, bagging [40] and random forests [41]. The survival trees are also commonly employed in ensemble methods like bagging [35, 42], boosting [33] and random forests $[34,43,44]$. An extensive review and discussion on the induction of survival trees and survival tree ensembles can be found in [45]. In this work the merits and limitations of these methods are discussed, along with the available computer software.

One of important aspects of using the survival analysis in medical sciences and bioinformatics is the necessity to have easily interpretable results. This ability is a crucial feature of survival trees and survival rules. Both approaches divide the observations into subgroups with different survivability characteristics. Importantly enough, they allow not only the attributes that have significant impact on the survival time to be identified, but also non-linear dependencies and interactions between the variables to be modelled.

While survival trees can be straightforwardly translated to survival rules, the algorithms used for induction of the latter directly from data have numerous advantages. Firstly, divide-and-conquer $(\mathrm{DnC})$ tree generation strategy forbids examples to be covered by multiple rules. Separate-and-conquer $(\mathrm{SnC})$ heuristics for rules induction lacks this limitation often leading to discovering stronger or completely new dependencies in the data. Secondly, generation of rules from the tree by following the path from the root to leafs results in condition redundancy. This is not the case in $\mathrm{SnC}$, as each rule is induced separately. The last feature is also useful when it is necessary to modify the generated rules so that they could better correspond to the domain knowledge. The SnCgenerated rules can be a preliminary set of hypotheses which is then verified by an analyst (domain expert). By adding or deleting elementary conditions from the rules, or modifying their ranges, the analyst can carry out different variants of the analysis. Consequently, adding new rules to the set does not interact with existing ones. The tree, in contrast, should be treated as a whole. Therefore, a change of a condition in a tree node involves the need to modify or re-calculate the conditions in all its child nodes.

\section{Objectives and outline}

The main goal of this paper is to present the separate-andconquer rule learning algorithm designed for survival data analysis and to verify its effectiveness on the variety of survival problems. In contrast to most of the aforementioned related work, we propose a more general solution rather than the case-study approach. Moreover, as opposed to $[9,10,12]$, the presented strategy does not require data pre-processing with the use of discretization methods. It is particularly important for the quality of survival analysis because discretization may cause the loss of information, and the final performance of the model may strongly depend on a selected discretization technique.

The key feature of our algorithm is the use of the separate-and-conquer strategy and log-rank statistical test for supervising the rule induction process. The log-rank test is aimed at detecting the most powerful and important factors affecting the expected survival time. Therefore, the resulting rule-based data models should be concise, easy to interpret by domain experts, and accurate in the survival time prediction. The use of the log ranktest requires neither the weight assignment to examples nor defining decision classes (e.g. event, non-event). All of these features distinguish the presented algorithm from the other approaches.

The efficiency of our rule-based framework for survival analysis was verified on a collection of 18 survival datasets describing a wide variety of real-life medical and biological problems. We compared our solution with the state-of-art survival trees algorithms.

In addition, we present the detailed analysis of rules sets for German Breast Cancer Study Group 2 [46], Bone Marrow Transplantation [47], Lung Adenocarcinoma [48], and Papillary Thyroid Carcinoma [49] datasets. The results show that the rule-based models generated by our algorithm are useful and can provide interesting information about the data, particularly when faced with the recent development of bioinformatics technologies.

The algorithm is available at http://www.adaa.polsl.pl/ software.html.

\section{Methods}

Let $D(A, T, \delta)$ be the survival dataset of $|D|$ observations (examples, instances). Each example is characterized by a set of covariates (attributes) $A=\left\{A_{1}, A_{2}, \ldots, A_{|A|}\right\}$, an observation time $T$, and a censoring status $\delta$. Therefore, $i$-th example can be represented as a vector $o_{i}=$ $\left(a_{i 1}, \ldots, a_{i|A|}, T_{i}, \delta_{i}\right)$. In the study we consider rightcensored data model which is the most common in the survival analysis. Consequently, $T_{i}$ denotes either the time of the observation for event-free examples $\left(\delta_{i}=0\right)$ or the time before the occurrence of an event $\left(\delta_{i}=1\right)$.

The LR-Rules algorithm returns a set of survival rules. A survival rule $r$ has the form:

$$
\text { IF } c_{1} \wedge c_{2} \wedge \ldots \wedge c_{n} \text { THEN } \hat{S}\left(T \mid c_{j}\right)
$$

The premise of the rule is a conjunction of conditions. If attribute $A_{j}$ is of nominal type, condition $c_{j}$ has the form $A_{j}=a_{j}$; if $A_{j}$ is numerical, $A_{j}<a_{j}$ or $A_{j} \geq a_{j}$ conditions are possible (with $a_{j}$ being an element of the $A_{j}$ domain). An observation is covered by the rule when it satisfies its premise. The conclusion of $r$ is an estimate $\hat{S}\left(T \mid c_{j}\right)$ of the survival function. Particularly, it is a Kaplan-Meier (KM) 
estimator [50] calculated on the basis of the instances covered by the rule, that is, satisfying all conditions $c_{j}(j=$ $1, \ldots, n)$.

The induction of survival rules in LR-Rules follows the separate-and-conquer heuristics. The algorithm adds rules iteratively to the initially empty set. Every learned rule has to cover at least mincov previously uncovered examples from the input dataset. The iteration continues until entire dataset becomes covered by the rule set. The pseudocode of the separate-and-conquer approach is presented in Algorithm 1.

The aim of the induction algorithm is to obtain rules of maximum quality. An extensive research on classification rules [21-23] showed that proper selection of a quality measure is of crucial importance for comprehensibility and performance of output model. In the survival analysis it is desirable for a rule to cover examples which survival distributions differ significantly from that of other instances. In presented algorithm, KM survival estimates of the examples covered and uncovered by the rule are derived from the data. A log-rank test statistics for those estimates is then used as a quality measure. The log-rank statistics is calculated as $x^{2} / y$ where:

$$
\begin{aligned}
& x=\sum_{t \in T_{c} \cup T_{u}}\left(d_{u}^{t}-\frac{r_{u}^{t}}{r_{c}^{t}+r_{u}^{t}} \cdot\left(d_{c}^{t}+d_{u}^{t}\right)\right) \\
& y=\sum_{t \in T_{c} \cup T_{u}} \frac{r_{c}^{t} \cdot r_{u}^{t} \cdot\left(d_{c}^{t}+d_{u}^{t}\right) \cdot\left(r_{c}^{t}+r_{u}^{t}-d_{c}^{t}-d_{u}^{t}\right)}{\left(r_{c}^{t}+r_{u}^{t}\right)^{2} \cdot\left(r_{c}^{t}+r_{u}^{t}-1\right)}
\end{aligned}
$$

$T_{c}$ and $T_{u}$ are sets of event times of observations covered and not covered by the rule, $d_{c}^{t}\left(d_{u}^{t}\right)$ is the number of covered (uncovered) observations which experienced an event at time $t$, and $r_{c}^{t}\left(r_{u}^{t}\right)$ is the number of covered (uncovered) instances at risk, that is, which are still observable at time $t$.

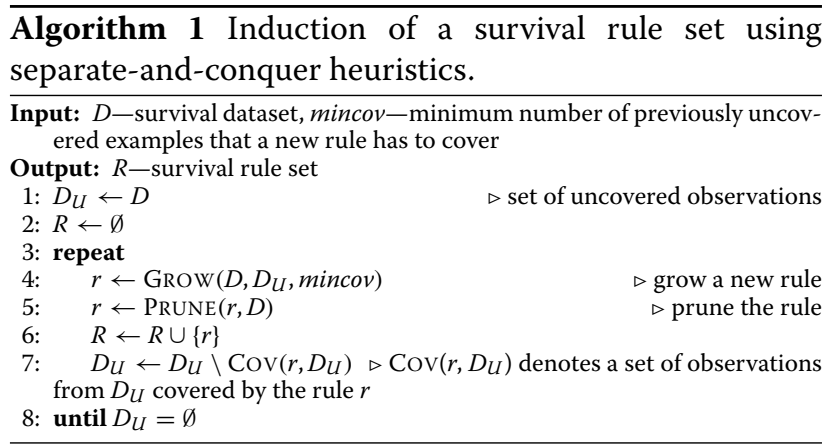

The induction of a rule is performed in two stages: growing and pruning. The former consists in greedy addition of elementary conditions to the initially empty rule premise (Algorithm 2). At each step, the algorithm searches exhaustively for the condition whose addition
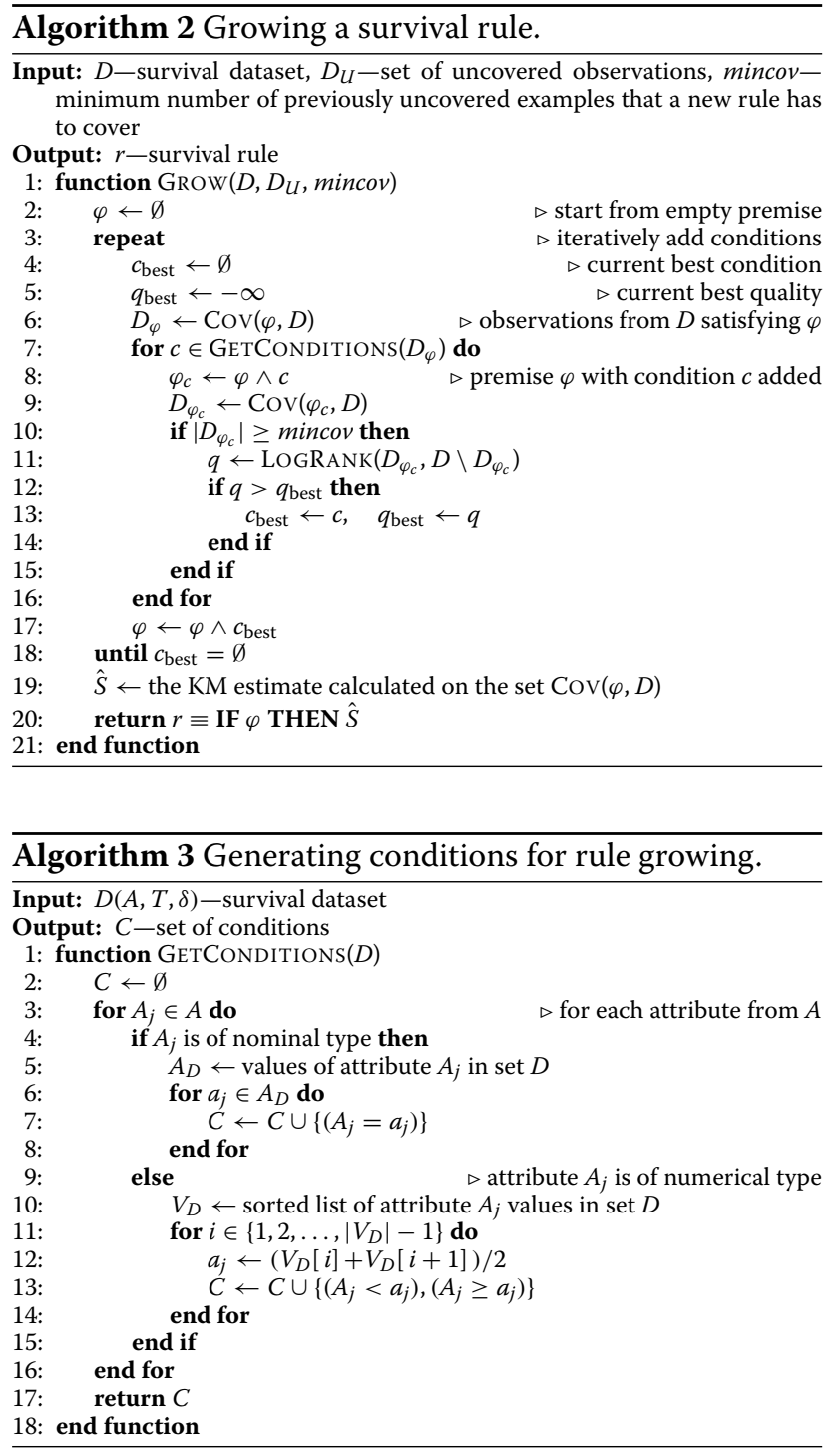

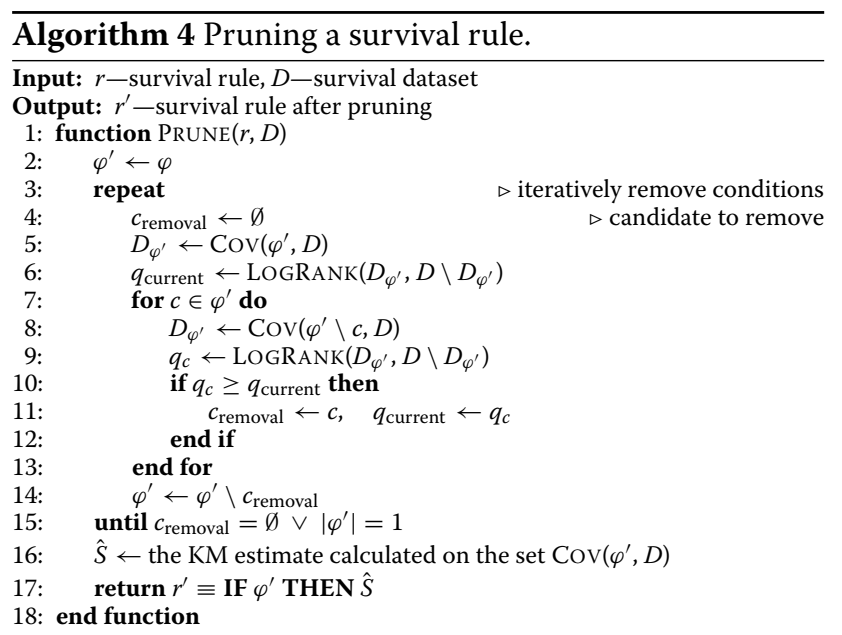


renders rule of the highest quality. If several conditions lead to the same value of the log-rank statistics, the one covering more examples is selected. The set of all the possible conditions which might be added to the rule is created on the basis of examples currently covered by the rule (Algorithm 3). In the case of nominal attributes, conditions in the form $A_{j}=a_{j}$ for all values $a_{j}$ from the attribute domain are considered. For continuous attributes, $A_{j}$ values that appear in the observations covered by the rule are sorted. Then, the possible split points $a_{j}$ are determined as arithmetic means of adjacent elements and conditions $A_{j}<a_{j}$ and $A_{j} \geq a_{j}$ are evaluated. Te prevent from generation of too specific rules, conditions whose addition would cause the rule to cover less than mincov previously uncovered examples are discarded. The growing stops when no conditions satisfying aforementioned criterion remain.

Growing stage is followed by pruning (Algorithm 4). The procedure iteratively removes conditions from the premise, each time making an elimination leading to the largest improvement in the quality. The procedure stops when no conditions can be deleted without decreasing the log-rank statistics or when rule contains only one condition.

Finally, for comprehensibility, the output rules are post processed by merging conditions based on the same numerical attributes. For example, the conjunction: $A_{i} \geq$ $x \wedge A_{i}<y$ is transformed into a single condition $A_{i} \in$ $\langle x, y)$.

Figure 1 illustrates the idea of rule growing supervised by the log-rank criterion. Let $r$ be the input rule with two

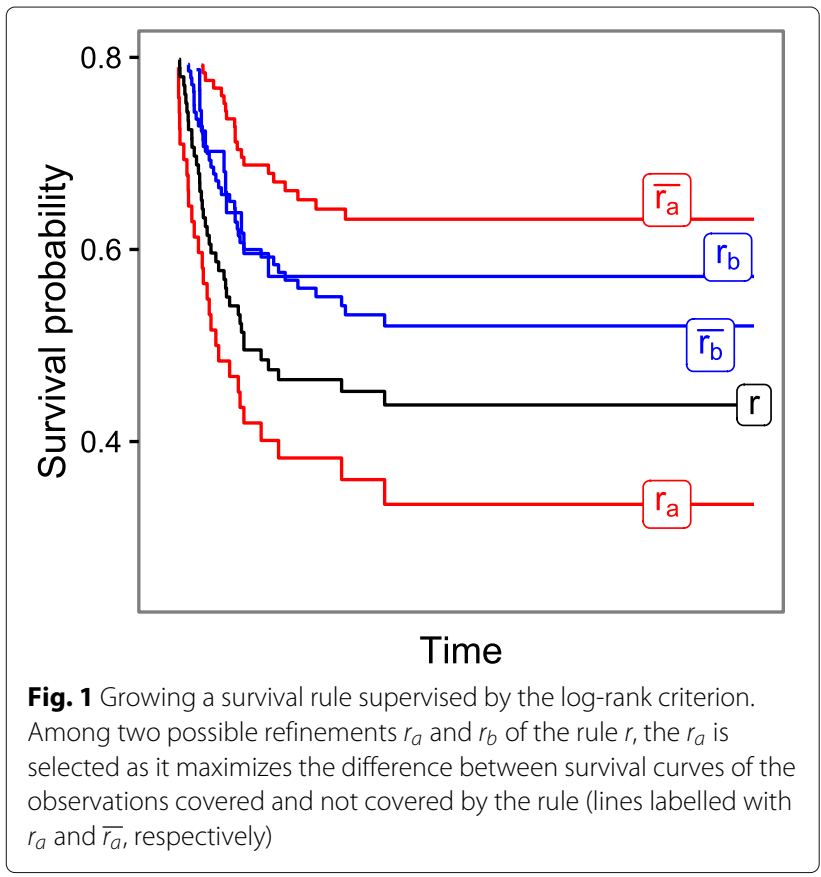

possible refinements $r_{a}$ and $r_{b}$. The figure shows the KM curves of all these rules. Additionally, the graph presents the survival curves of the observations not covered by the rules $r_{a}$ and $r_{b}$, labelled with $\overline{r_{a}}$ and $\overline{r_{b}}$, respectively. The log-rank statistics calculated for the rule $r_{a}\left(r_{b}\right)$ reflects difference between survival curves labelled with $r_{a}\left(r_{b}\right)$ and $\overline{r_{a}}\left(\overline{r_{b}}\right)$. The difference between $r_{a}$ and $\overline{r_{a}}$ is greater than for the pair $r_{b}-\overline{r_{b}}$. Therefore, the refinement $r_{a}$ of the rule $r$ better discriminates observations according to the survival rate, thus it is selected as the current best form of the rule which is expanded with new conditions in the subsequent iterations.

In order to deal with missing attribute values, LRRules employs an ignored value strategy in which rules are built based only on known values of observations. It is performed straightforwardly by skipping missing values during search of possible conditions. The observation having a missing value of an attribute tested by the rule is considered to be uncovered by this rule. In contrast to imputation methods [51], this strategy does not require any additional computations and, as was shown in [52], it performs similarly to more advanced and computationally expensive approaches to handling missing values.

A valuable property of LR-Rules is also the ability to handle datasets with weighted observations. In this case, the value of log-rank test is calculated on the basis of weights and mincov parameter indicate the sum of observations weights to be covered by a newly generated rule.

The learned rule set can be applied for an estimation of the survival function of new observations based on the values taken by their covariates. The estimation is performed by rules covering given observation. If observation is not covered by any of the rules then it has assigned the default survival estimate computed on the entire training set. Otherwise, final survival estimate is calculated as an average of survival estimates of all rules covering the observation (see Fig. 2 for an example).

\section{Results and discussion Experimental setting}

The LR-Rules algorithm was investigated on 18 sets listed in Table 1 using 10-fold stratified cross-validation repeated ten times for each set. The stratification of survival data was performed according to the censoring status, that is, the proportion of events to censored observations in each fold was the same as in the entire training set. Additionally, the detailed analysis of survival rules was performed on four selected sets. These were GBSG2 (German Breast Cancer Study Group 2) [53], BMT-Ch (Bone Marrow Transplantation - Children) [20, 47], LAC (Lung Adenocarcinoma) [48], and PTC (Papillary Thyroid Carcinoma) [49].

GBSG2 is a well-known dataset which describes patients with primary node positive breast cancer. It was used, 


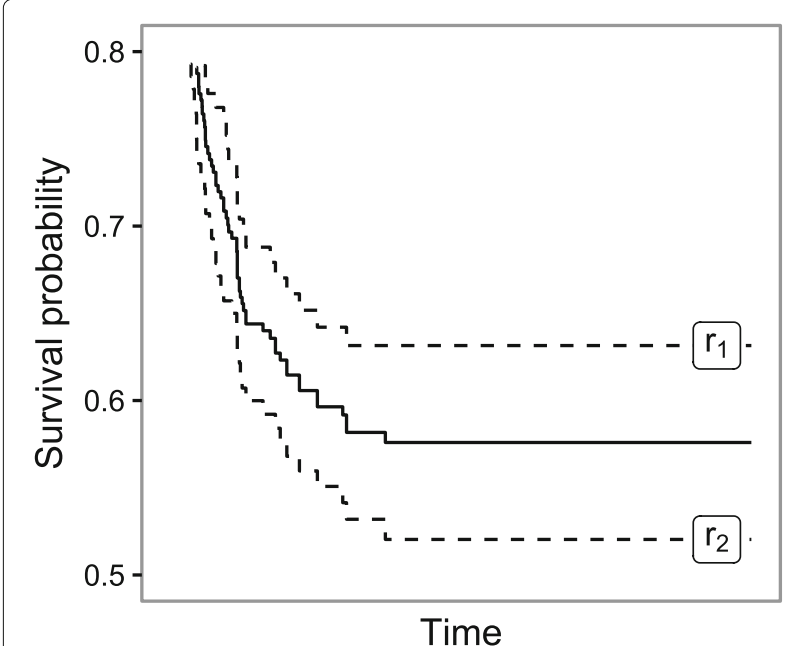

Fig. 2 Averaging survival curves. When the observation is covered by multiple rules ( $r_{1}$ and $r_{2}$ in this case), its survival function (solid line) is obtained as an average of the rule functions (dashed lines)

inter alia, in [12, 31, 39] to test different modeling techniques. Each observation is described by the following attributes: hormonal therapy (horTh), age, menopausal status (menostat), tumour size (tsize), tumour grade (tgrade), number of positive nodes (pnodes), progesterone

Table 1 The characteristics of 18 sets used in the experimental studies: the number of observations (\#obs), the number of conditional attributes (\#att), the percentage of missing values $(\% \mathrm{mv})$, the percentage of censored observations (\%cs), and the research subject

\begin{tabular}{llllll}
\hline Set & \#obs & \#att & $\% m v$ & $\% c s$ & Subject of research \\
\hline actg320 [63] & 1151 & 11 & 0 & 92 & HIV-positive patients \\
BMT-Ch [47] & 187 & 37 & 1 & 55 & Bone marrow transplant \\
cancer [64] & 228 & 7 & 4 & 28 & Advanced lung cancer \\
follic [65] & 541 & 4 & 0 & 36 & Follicular lymphoma \\
GBSG2 [53] & 686 & 8 & 0 & 56 & Breast cancer \\
hd [65] & 865 & 6 & 0 & 51 & Hodgkin's disease \\
LAC [48] & 86 & 113 & 0 & 72 & Lung adenocarcinoma \\
lung [66] & 1032 & 7 & 3 & 26 & Early lung cancer \\
Melanoma [67] & 205 & 7 & 0 & 65 & Malignant melanoma \\
mgus [68] & 241 & 9 & 20 & 24 & Monoclonal gammopathy \\
PTC [49] & 421 & 24 & 41 & 93 & Papillary thyroid carcinoma \\
pbc [69] & 418 & 17 & 15 & 61 & Primary biliary cirrhosis \\
std [70] & 877 & 21 & 0 & 60 & Sexually-trans. diseases \\
uis [63] & 575 & 13 & 0 & 19 & Drug addiction treatment \\
wcgs [71] & 3154 & 10 & $<1$ & 92 & Coronary artery disease \\
whas1 [63] & 481 & 7 & 0 & 48 & Myocardial infarction ed1 \\
whas500 [63] & 500 & 13 & 0 & 57 & Myocardial infarction ed2 \\
zinc [72] & 431 & 55 & 57 & 81 & Esophageal cancer \\
\hline
\end{tabular}

receptor (progrec), estrogen receptor (estrec). An event in survival analysis is cancer recurrence.

BMT-Ch describes 187 patients (75 females and 112 males) at the age of 0.6 to 20.2 years (median 9.6) admitted to the Department of Pediatric Bone Marrow Transplantation, Oncology and Hematology, Wrocław Medical University, Poland. Disease spectrum included 155 malignant disorders (i.a. 67 patients with acute lymphoblastic leukemia, 33 with acute myelogenous leukemia, 25 with chronic myelogenous leukemia, 18 with myelodysplastic syndrome) and 32 nonmalignant cases (i.a. 13 patients with severe aplastic anemia, 5 with Fanconi anemia, 4 with X-linked adrenoleukodystrophy). The procedure of unmanipulated allogeneic unrelated donor hematopoietic stem cell transplantation was performed in each case, according to the European protocols or the guidelines of the European Blood and Marrow Transplant Inborn Errors Working Party with worldwide accepted modifications based on disease and/or patient's condition status prior transplantation. Each patient was characterized by a set of 42 conditional attributes. Table 2 presents interpretations of selected ones. Patient's death is considered as an event.

LAC dataset concerns gene expression profiles of 86 lung cancer patients [48]. Expressions were measured with Affymetrix hu6800 microarrays (7129 probe sets) and normalized from raw .CEL files by RMAExpress. In the experiments we considered 100 genes with greatest effect on survival rate according to Beer et al. [48]. Due to name discrepancies, three genes were excluded from the investigation as they did not map to any probe. On the other hand, some genes had multiple probes assigned. As a result, LAC dataset contains 113 conditional attributes with patient's death being considered as an event.

PTC gathers information about 492 papillary thyroid cancer patients. They are characterized by clinical as well as genome-related features like single nucleotide polymorphisms (SNP), copy number alterations (CNA), gene expressions determined with RNA-seq, DNA methylation, protein expressions obtained by reverse phase protein arrays (RPPA), etc. Data table available at [54] was processed by filtering out patients with missing information about survival status or survival time. As we wanted to focus this study on the genetic background of thyroid cancer, corresponding features were selected for further analysis (Table 3). We assumed recurrence of a cancer to be an event in the survival analysis.

The results of the LR-Rules algorithm were compared with results achieved by the KM estimator, our earlier CW-Rules algorithm [20], and two implementations of survival trees (CTREE, RPART). The CTREE algorithm [39] builds model from survival data using a splitting criterion based on the log-rank statistic. The RPART algorithm [55] fits time variable into exponential model, and then 
Table 2 Selected conditional attributes of BMT-Ch (Bone Marrow Transplantation) dataset

\begin{tabular}{|c|c|}
\hline Name & Description \\
\hline RecipientRh & $\begin{array}{l}\text { Presence of the Rh factor on recipient's red } \\
\text { blood cells }\end{array}$ \\
\hline RecipientAge & $\begin{array}{l}\text { Age of the recipient of hematopoietic stem } \\
\text { cells at the time of transplantation }\end{array}$ \\
\hline RecipientBodyMass & $\begin{array}{l}\text { Body mass of the recipient of hematopoietic } \\
\text { stem cells at the time of transplantation }\end{array}$ \\
\hline CMV_status & $\begin{array}{l}\text { Serological compatibility of the donor and } \\
\text { the recipient of hematopoietic stem cells } \\
\text { according to cytomegalovirus infection } \\
\text { prior to transplantation }\end{array}$ \\
\hline Recipient $A B O$ & $\begin{array}{l}\text { ABO blood group of the recipient of } \\
\text { hematopoietic stem cells }\end{array}$ \\
\hline DonorABO & $\begin{array}{l}\text { ABO blood group of the donor of } \\
\text { hematopoietic stem cells }\end{array}$ \\
\hline ABOmatch & $\begin{array}{l}\text { Compatibility of the donor and the recipient } \\
\text { of hematopoietic stem cells according to } \\
\text { ABO blood group }\end{array}$ \\
\hline DonorAge & $\begin{array}{l}\text { Age of the donor at the time of } \\
\text { hematopoietic stem cells apheresis }\end{array}$ \\
\hline HLAmatchCompatibility & $\begin{array}{l}\text { Compatibility of antigens of the main } \\
\text { histocompatibility complex of the donor } \\
\text { and the recipient of hematopoietic stem } \\
\text { cells ( } 10 / 10,9 / 10,8 / 10,7 / 10 \text { allele/antigens) } \\
\text { according to ALL international BFM SCT } \\
2008 \text { criteria }\end{array}$ \\
\hline Relapse & Reoccurrence of the disease \\
\hline GVHD_III_IV & $\begin{array}{l}\text { Development of acute graft versus host } \\
\text { disease stage III or IV }\end{array}$ \\
\hline extcGvHD & Extensive chronic graft versus host disease \\
\hline CD34 $\left(10^{6} / \mathrm{kg}\right)$ & $\begin{array}{l}\text { CD34+ cell dose per kg of recipient body } \\
\text { weight }\end{array}$ \\
\hline $\operatorname{CD} 3\left(10^{8} / \mathrm{kg}\right)$ & $\begin{array}{l}\text { CD3+ cell dose per kg of recipient body } \\
\text { weight }\end{array}$ \\
\hline$C D 3 / C D 34$ & CD3+ cell to CD34+ cell ratio \\
\hline
\end{tabular}

it applies Poisson regression to such modified data. It leads to method equivalent to the deviance residual-based approach of LeBlanc and Crowley [16].

The performance of rule sets was evaluated with a use of the integrated Brier score (IBS) [56, 57]. The Brier score at time $T^{\star}$ for $\mathrm{i}$-th observation is given by:

$$
B S_{i}\left(T^{\star}\right)= \begin{cases}\frac{1}{\hat{G}\left(T_{i}\right)} \cdot\left[0-\hat{S}\left(T^{\star}\right)\right]^{2} & \text { if } T_{i} \leq T^{\star}, \delta_{i}=1 \\ \frac{1}{\hat{G}\left(T^{\star}\right)} \cdot\left[1-\hat{S}\left(T^{\star}\right)\right]^{2} & \text { if } T_{i}>T^{\star} \\ 0 & \text { in other cases }\end{cases}
$$

The Brier score $B S_{i}\left(T^{\star}\right)$ represents the squared difference between true event status at time $T^{\star}$ and predicted event status $\hat{S}\left(T^{\star}\right)$ at that time. The true event status for $\mathrm{i}$-th observation is equal to 0 if an event occurred for this observation before or at the time $T^{\star}$, and it is equal to 1 if
Table 3 Selected conditional attributes of PTC (Papillary Thyroid Carcinoma) dataset

\begin{tabular}{ll}
\hline Name & Description \\
\hline BRAFV600ERAFClass & $\begin{array}{l}\text { Flag indicating if tumor is driven by BRAF or } \\
\text { RAS genes }\end{array}$ \\
BRAFV600E_RAS_score & $\begin{array}{l}\text { Continuous score from }(-1,1) \text { interval } \\
\text { describing to what extent a tumor } \\
\text { expression profile resembles BRAF- or } \\
\text { RAS-mutant profiles }\end{array}$ \\
mRNA_cluster_number & $\begin{array}{l}\text { Number of mRNA expression cluster (1-5) } \\
\text { miRNA_cluster_number }\end{array}$ \\
RPPA_cluster_number & $\begin{array}{l}\text { Number of microRNA expression cluster } \\
\text { meth_cluster }\end{array}$ \\
Arm_SCNA_cluster & $\begin{array}{l}\text { DNA methylation pattern (one of four) } \\
\text { Chromosomal arm-level copy number } \\
\text { alterations pattern (one of four) }\end{array}$ \\
nmut_APOBEC & $\begin{array}{l}\text { Mutation density (mutations/Mb) associated } \\
\text { with APOBEC cytidine deaminases }\end{array}$ \\
nmut_CpGT & $\begin{array}{l}\text { Mutation density (mutations/Mb) of CpG } \\
\text { islands } \\
\text { Gender }\end{array}$ \\
person_gender & Race (Black/White/Asian/American Indian) \\
race_category & Ethnicity (Hispanic/Non-Hispanic) \\
ethnicity_category &
\end{tabular}

survival time $T_{i}$ of the observation is greater than $T^{\star}$. The censoring is taken into account by weighting the squared differences by the inverse of the estimate $\hat{G}$ of the censoring survival function. The $\hat{G}$ estimate is calculated as the KM estimator based on training observations with censoring status set to $(1-\delta)$. If observation was censored before time $T^{\star}$ then its weight is equal to 0 . However, such observations have indirect contribution to final score because they are considered in calculation of $\hat{G}$ estimate.

The IBS summarizes the prediction error over all $n$ observations and over all times in a test set:

$$
I B S=\frac{1}{\max T_{i}} \int_{0}^{\max T_{i}} B S\left(T^{\star}\right) d T^{\star}
$$

where

$$
B S\left(T^{\star}\right)=\frac{1}{n} \sum_{i=1}^{n} B S_{i}\left(T^{\star}\right)
$$

Lower IBS values correspond to better prediction accuracy.

In the experimental study, the algorithms were compared on multiple datasets with the use of statistical tests recommend by Demšar [58]. For comparison of two algorithms on multiple datasets we used the Wilcoxon signed rank test, while the comparisons of all algorithms with each other were preformed with a use of the Friedman test followed by the post-hoc Nemenyi test. 


\section{Experimental evaluation}

The first experimental step was to investigate the influence of mincov parameter on the results of the LR-Rules algorithm. This parameter specifies the minimum number of uncovered observations that must be covered by a newly generated rule during the growing phase. The minimum value of this parameter is 1 , which corresponds to the case when each induced rule must cover at least one yet uncovered example. The greater the value of mincov, the higher is the coverage of resulting rules. This decreases the cardinality of the final rule set.

In the study, mincov values ranging from 1 to 7 were examined. The upper bound of seven was selected as this is a default value of the minbucket parameter, which defines the minimum number of observations in the leaves of CTREE and RPART trees. Detailed results, i.e., Brier scores and numbers of rules for different mincov values are presented in Additional file 1: Tables S1 and S2.

The analysis of mincov effect on IBS with a use of Friedman test, revealed that at least one of the investigated parameter values generated models of significantly different accuracy than the others ( $p$-value of 0.0478 ). However, the results of the Nemenyi's post-hoc test (summarized in Additional file 1: Figure S1 as a critical difference diagram) showed no statistical significance at 0.05 level.

The different situation was in the case of the size of resulting rule sets. As presented in Fig. 3, increasing mincov parameter caused noticeable reduction in the number of rules. Importantly enough, the greater the initial model, the larger decrease was observed. The comparison of parameter values with a use of the Friedman test rejected the null hypothesis about all parameter values generating same number of rules with $p$-value close to zero. A summary of the Nemenyi post-hoc test (Additional file 1: Figure S2) revealed the lack of significance only within groups of three neighbouring mincov values. The strong dependency between mincov and the model size was also confirmed statistically: the Pearson's correlation between the parameter value and the rank was close to -1.0 .

Setting mincov parameter to 7 resulted in the most compact models: for the majority of survival datasets containing hundreds of observations, the algorithm generated less than eight rules. For this reason, and due to lack of significant effect of the parameter on the accuracy, 7 was set as the default mincov value in LR-Rules and was used in further experiments, unless specified otherwise.

The next part of the study was to compare LR-Rules to CW-Rules, CTREE, RPART, and the KM estimator in terms of the accuracy and the model size. The results for particular datasets are presented in Fig. 4 as bubbles with horizontal coordinates corresponding to IBS (lower = better) and diameter proportional to the logarithm of the number of rules. The results in the numerical form can be found in Additional file 1: Tables S3 and S4.

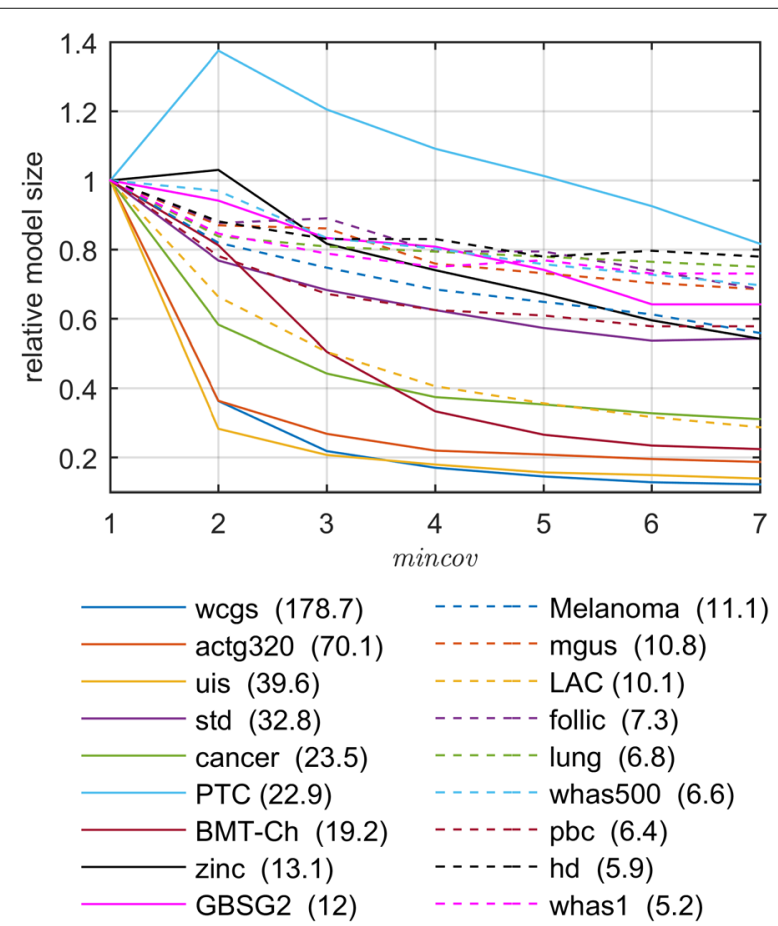

Fig. 3 Influence of the mincov parameter on the LR-Rules model size. The model size for each dataset is defined as the number of rules normalized by the number of rules for mincov $=1$ (given in the legend)

The Friedman test showed statistically significant differences between the LR-Rules, CW-Rules, CTREE, RPART and $K M$ algorithms in terms of the IBS criterion ( $p$-value $\left.<10^{-4}\right)$. The visualization of Nemenyi's post-hoc test at the 0.05 significance level is presented in Fig. 5. LR-Rules was in the group of three best algorithms together with the CW-Rules and CTREE. The worst results were obtained by the KM estimator. Interestingly, the Nemenyi's test indicated no difference between KM, RPART and LRRules. Nevertheless, as this test is often too conservative to show the difference [59], an additional comparison between LR-Rules and the competitors was carried out using the Wilcoxon test with the Finner correction [60]. The test showed our algorithm to be superior to the KM estimator in terms of IBS ( $\left.p_{\text {corrected }}=0.0062\right)$. In contrast, the comparison with CTREE and RPART revealed lack of significance (both uncorrected and corrected $p$-values were noticeably greater than 0.05$)$. CW-Rules achieved lower prediction error on the investigated data than LRRules (corrected $p$-value equaled to 0.0051).

As Additional file 1: Table S4 shows, superior accuracy of CW-Rules was obtained at the cost of the model size: for all analyzed datasets it generated several times greater rule sets than other methods. This was confirmed by the statistical analysis. While LR-Rules, CTREE, and RPART generated models of similar complexity (lack of 


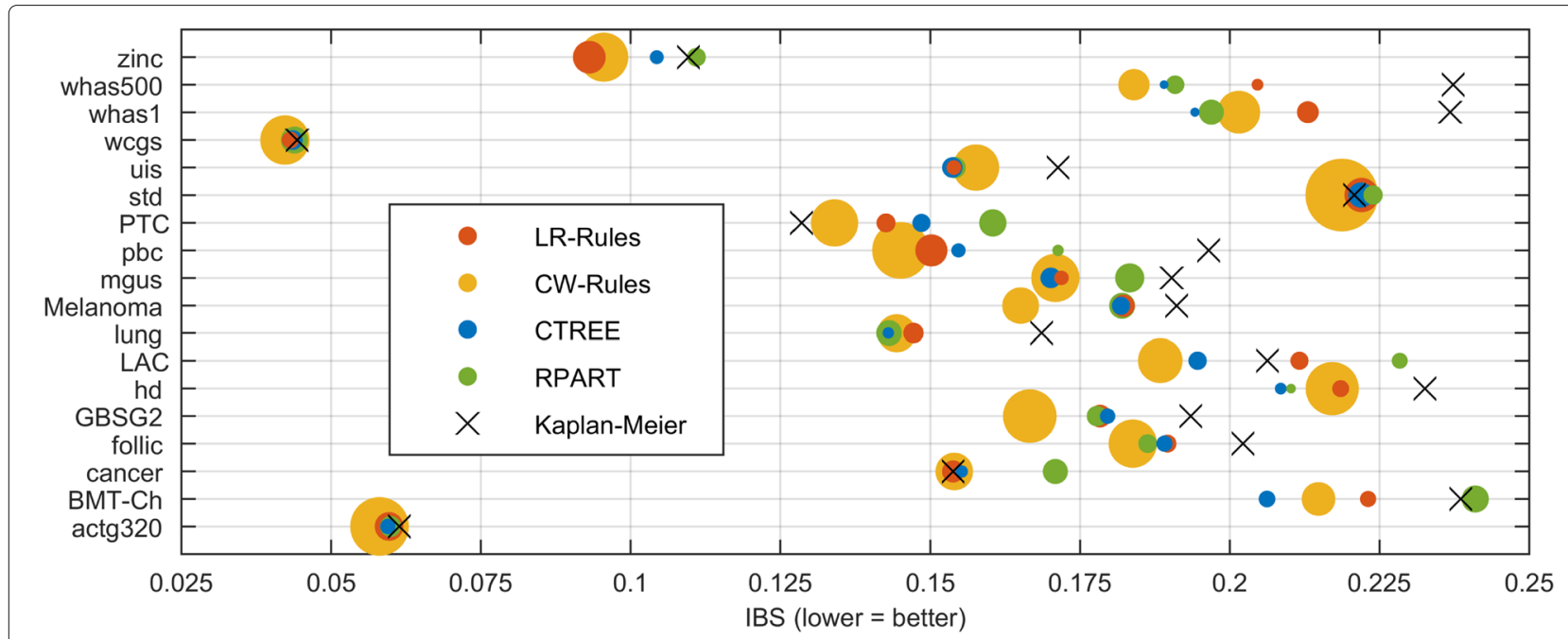

Fig. 4 Comparison of the algorithms on the investigated datasets. Horizontal axis corresponds to the prediction accuracy (IBS), bubble diameters are proportional to the logarithm of the number of rules

significance at 0.05 level), CW-Rules induced significantly more rules (Additional file 1: Figure S3).

Table 4 provides detailed characteristics of the models generated by LR-Rules. The output rules usually contained from 1 to 7 elementary conditions, but majority of them had at most 3 conditions. Each of rules covered on average $36 \%$ of the observations from the training set. Importantly, the greater the number of rules in a set, the lower the coverage: the Pearson correlation coefficient between those variables equaled to -0.9135 . The significance of rules was assessed statistically by performing log-rank test between Kaplan-Meier estimators of observations covered and uncovered by investigated rule. To control false discovery rate, the Benjamini-Hochberg correction was applied [61]. As it is shown in Table 4, the percentage of statistically significant rules at 0.05 level was close to $100 \%$.

\section{Case studies}

In order to demonstrate the rules induced by the presented algorithm, the detailed analysis of GBSG2, BMTCh, LAC, and PTC was performed. To obtain the most comprehensible models for the investigated datasets, mincov parameter was set to $3,5,7$, and 12 , respectively.
The rule set induced by the algorithm for the whole GBSG2 dataset consisted of 10 rules. Four of them are presented below:

R1: progrec $\geq 108.0$

R2: pnodes $<5.5 \wedge$ progrec $\geq 16.5 \wedge$ age $\geq 39.5$

R3: pnodes $\geq 4.5 \wedge$ progrec $<23 \wedge$ age $\in[41.5,59.5)$

$\wedge$ estrec $\in[0.5,37.0)$

R4: pnodes $\geq 4.5 \wedge$ progrec $<28.5$

The KM survival curves for observations covered by the R1-R4 rules are presented in Fig. 6a. The graph additionally includes a default curve representing the KM estimate for the entire GBSG2 dataset. The significant difference can be observed between the survival estimates determined by R1-R2 rules, which are above the default estimate, and R3-R4, which are placed below. Neither of the 10 induced rules had horTh attribute, indicating that the patient was a subject to the hormonal therapy. This result is consistent with the conclusions of the work [46], stating that: No significant difference in recurrence-free survival was observed with respect to hormonal therapy.

The rule set induced by the algorithm for the entire BMT-Ch data consisted of 7 rules. The motivation of this study was to identify the most important factors

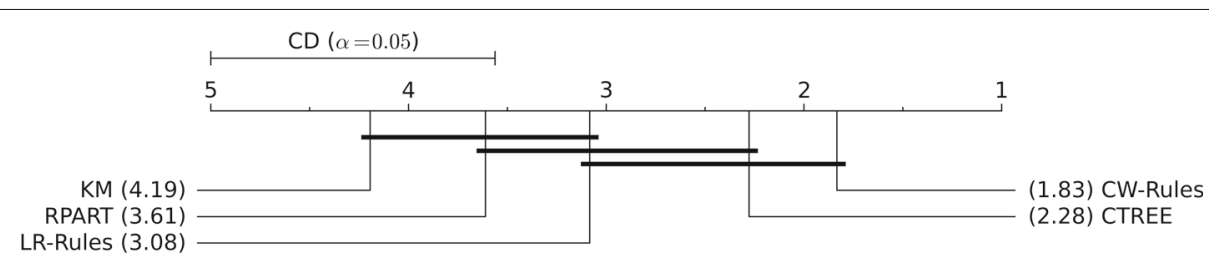

Fig. 5 Statistical analysis of the prediction accuracy. Critical difference diagram comparing LR-Rules, CW-Rules, CTREE, RPART algorithms, and the KM estimator in terms of the integrated Brier score (IBS) at the significance level 0.05 over 18 datasets. Average ranks are shown in parentheses (lower $=$ better). The groups of algorithms which are not significantly different are connected with bold lines 
Table 4 The characteristics of rule sets generated by LR-Rules: the value of the integrated Brier score (IBS), the number of generated rules (\#rules), the average rule length, the average rule coverage (\%cov), a percentage of significant rules ( $p$-value of log-rank test with FDR adjustment below 0.05; \%sign)

\begin{tabular}{llllll}
\hline Dataset & IBS & \#rules & Length & \%cov & \%sign \\
\hline actg320 & 0.0597 & 13.1 & 3.7 & 28 & 99.18 \\
BMT-Ch & 0.2231 & 4.3 & 3.3 & 48 & 98.40 \\
cancer & 0.1538 & 7.3 & 3.4 & 40 & 97.35 \\
follic & 0.1896 & 5.0 & 1.8 & 38 & 99.32 \\
GBSG2 & 0.1783 & 7.7 & 2.7 & 36 & 99.75 \\
hd & 0.2185 & 4.6 & 1.3 & 44 & 99.43 \\
LAC & 0.2116 & 2.8 & 6.8 & 41 & 100.00 \\
lung & 0.1472 & 5.1 & 1.0 & 45 & 100.00 \\
Melanoma & 0.1823 & 6.2 & 2.6 & 33 & 99.69 \\
mgus & 0.1719 & 7.4 & 3.2 & 34 & 99.70 \\
PTC & 0.1426 & 18.7 & 3.0 & 22 & 79.69 \\
pbc & 0.1502 & 3.7 & 1.1 & 45 & 100.00 \\
std & 0.2220 & 17.8 & 6.5 & 25 & 98.74 \\
uis & 0.1539 & 5.5 & 1.2 & 41 & 100.00 \\
wcgs & 0.0432 & 21.8 & 5.2 & 19 & 98.95 \\
whas1 & 0.2130 & 3.8 & 1.3 & 43 & 100.00 \\
whas500 & 0.2046 & 4.6 & 1.2 & 40 & 100.00 \\
zinc & 0.0931 & 7.1 & 2.5 & 31 & 98.42 \\
\hline
\end{tabular}

influencing the success or failure of the transplantation procedure. In particular, verification of the research hypothesis that increased dosage of CD34+ cells $/ \mathrm{kg}$ extends overall survival time without simultaneous occurrence of undesirable events affecting patients' quality of life $[11,47]$. Four of the induced rules are presented below:

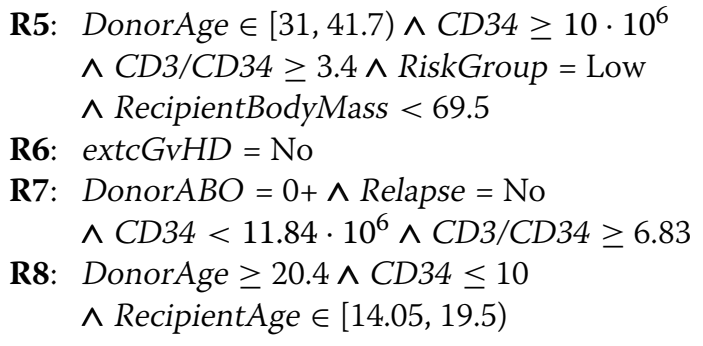

Figure $6 \mathrm{~b}$ presents the KM survival curves for observations covered by the R5-R8 rules, as well as the default estimate for the entire dataset. As in the previous case, the R5-R6 curves are above the default estimate, while R7-R8 are below.

The CD34 attribute occurred often in the induced rules. It can be seen that lower doses of the CD34 cells affected the shorter survival time, while higher doses increased this time. In the paper [47] the impact of CD34 doses on the overall survival time was analyzed by dividing the value of $C D 34$ into two intervals: $\leq 10$ and $>10$. The rules induced by the proposed algorithm are consistent with [47] and they additionally clarify the conditions under which the doses of CD34 are even more important for the survival time. It should also be noted that the rule R6 states that patients without a chronic form of GvHD are characterized by the shorter survival time. This is also consistent with medical knowledge.

Another experiment concerned LAC dataset for which presented algorithm induced 3 survival rules. Each of them incorporate expression levels of 8 up to 10 genes. The analysis of Fig. 6c confirms that obtained rules effectively distinguish patients's survival rates on the basis of their expression profiles. The example survival rule has the following form:

$$
\begin{aligned}
\text { R1: } & S L C 2 O A 1<10.2 \wedge I T G A 2<8.7 \\
& \wedge V E G F<10.5 \wedge \text { REG } 1 A<10.8 \\
& \wedge S L C 2 A 1<8.9 \wedge \text { SCGB2A2<8.1 } \\
& \wedge S 100 P \geq 8.7 \wedge A T P 2 B 1<9.9
\end{aligned}
$$

When applied on PTC dataset, LR-Rules generated 16 rules. Most common attributes were BRAFV600E_RAS_score and nmut_CpGT (11 and 9 occurrences, respectively) which had been previously associated with thyroid cancer development [49]. Selected survival rules are presented below.

R1: $n$ mut_CPGT $\geq 4.5$ $\wedge$ mRNA_cluster_number $=5$

$\wedge$ BRAFV600E_RAS_score $\in(-0.976,-0.698)$

R2: RPPA_cluster_number $=3$

$\wedge$ mRNA_cluster_number $=5$

$\wedge$ BRAFV600E_RAS_score $<-0.868$

R4: meth_cluster $=$ classical 2

$\wedge$ Arm_SCNA_cluster $=$ Quiet

$\wedge$ miRNA_cluster_number $=6$

$\wedge$ nmut_CPGT $<5.5$

$\wedge$ BRAFV600E_RAS_score $\in(-0.974,-0.889)$

R8: Arm_SCNA_Cluster $=$ Quiet

$\wedge$ nmut_CPGT $\geq 1.5$

$\wedge$ BRAFV600E_RAS_score $\geq 0.573$

R14: $n$ mut_CPGT $<6.5$
$\wedge$ BRAFV600E_RAS_score $\in[0.676,0.919)$

As Fig. 6d shows, the corresponding survival curves differ noticeably. Obtained rules model complex relationships between attributes and their influence on the survival time. For instance, $\mathrm{BRAF}^{\mathrm{V} 600 \mathrm{E}}$ and RAS were proven to be driver genes in many cancers including PTC [62]. Nevertheless, the effect of mutations in those genes on probability of recurrence is altered by other attributes. Particularly, BRAF-like tumors (those characterized by low values of BRAFV600E_RAS_score) may differ significantly in survival rate (compare R1, R2, and R4 rules). The same situation was in the case of RAS-driven cancers (rules R8 and R14). 

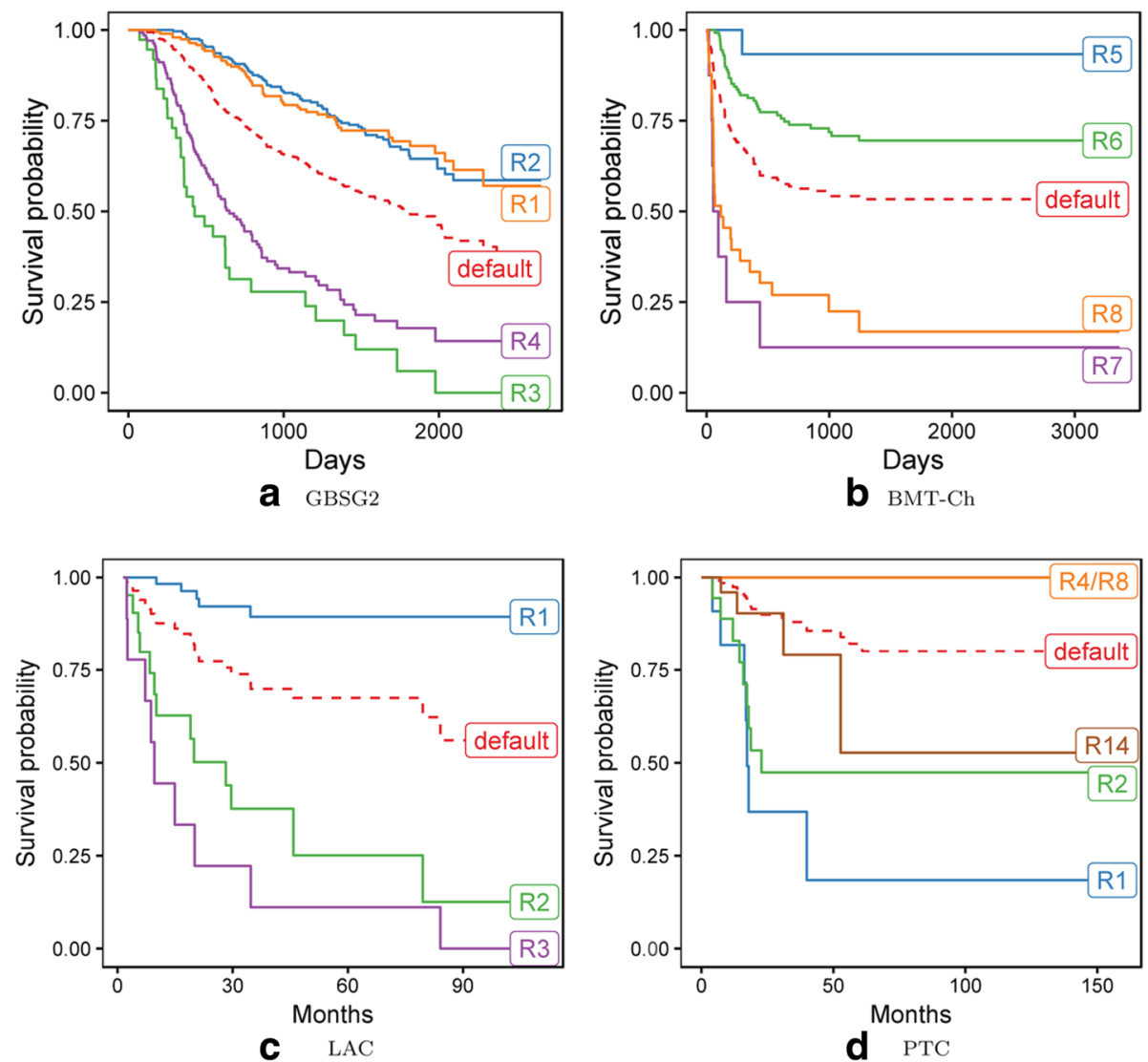

Fig. 6 Analysis of the rules induced for GBSG2 (a), BMT-Ch (b), LAC (c), and PTC (d) datasets. The charts demonstrate the Kaplan-Meier survival curves for the observations covered by selected rules. The dashed (default) curves represent the KM estimates of the entire datasets

\section{Conclusions}

The experiments confirmed LR-Rules to perform significantly better than the KM estimator and similarly to survival trees CTREE and RPART in terms of prediction error. The comparison of LR-Rules and CW-Rules shows that the latter tends to get lower IBS values than our algorithm. This, however, is obtained at the cost of model complexity: CW-Rules always generated more rules than the competitors. In contrast, LR-Rules produces compact sets of rules of similar size as the tree models CTREE and RPART.

Superior performance and model comprehensibility make LR-Rules an effective alternative or a complement to survival trees, such as CTREE and RPART. Although every tree may be presented as a set of rules, the divideand-conquer strategy used for tree construction usually leads to different rule sets than those generated by LR-Rules employing separate-and-conquer approach. In accordance with the strategy of tree building, every observation can be covered by exactly one rule, while the covering approach used by LR-Rules allows observations to be covered by multiple rules. The absence of this restrictive limitation in the presented algorithm may lead to the discovery of new or stronger patterns than those found by survival trees.

A characteristic feature of rule sets derived form a tree is the redundancy of conditions, particularly of the initial one that appears in every rule. In contrast, LR-Rules has the ability to induce rules with unique attributes. For example, when analysing BMT-Ch set, our algorithm generated a rule with only one condition: "extcGvHD = No". In order to derive such a rule from a tree, an attribute extcGvHD would have to appear in the root, and thus, all the other rules would have to also take it into account.

An important advantage of the rule sets returned by the LR-Rules algorithm is also the fact that each rule can be considered independently from the others. This feature can be useful if modification of the generated rules is required, for example, in order to reflect the domain knowledge in a better way. The rules automatically generated by LR-Rules may constitute an initial set of hypotheses for the analyst. The expert, by adding/removing the rule conditions or by modifying their ranges, is allowed to carry out the different variants of the analysis. New rules can also be added to an existing set straightforwardly. In contrast, trees have to be treated as a whole 
in order to preserve the disjointed nature of the rules. Thus, the change of a condition in a node involves the need for modification of the conditions in all of its child nodes. Similarly, adding new rule to the tree requires its reconstruction.

We expect that the importance of survival analysis in medicine and biology will increase due to dissamination of high througput sequencing. Establishing how patients' survival rate is affected by the presence of genetic variants, DNA methylation, or expressions of genes, microRNAs, and proteins will become of centeral interest. The application of LR-Rules on LAC and PTC datasets revealed interesting dependencies between genome/transcriptome/proteome-related features and their influence on the survival.

One of the greatest challenges to be faced when analyzing bioinformatics data is excessive dimensionality. High throughput technologies are able to produce hundreds of thousands of raw attributes which is prohibitive for machine learning strategies. Therefore, the application of all investigated survival analysis algorithms including LR-Rules has to be preceded by dimensionality reduction phase, i.e., construction, extraction and/or selection of features.

\section{Additional file}

Additional file 1: Supplementary file. Table S1: IBS scores for different mincov values, Table S2: Number of rules for different mincov values, Table S3: IBS scores for different algorithms, Table S4: Number of rules for different algorithms, Figure S1: CD-diagrams comparing different mincov values with respect to IBS, Figure S2: CD-diagrams comparing different mincor values with respect to the number of rules, Figure $\mathbf{S 3}$ : CD-diagrams comparing algorithms with respect to the number of rules. (PDF $875 \mathrm{~kb}$ )

\section{Abbreviations}

BS: Brier score; CD diagram: Critical difference diagram; CNA: Copy number alteration; DnC: Divide-and-conquer; GvHD: Graft versus host disease; IBS: Integrated Brier score; KM: Kaplan-Meier; LAD: Logical analysis of data; PI: Prognostic index; RPPA: Reverse phase protein arrays; SnC:

Separate-and-conquer; SNP: Single nucleotide polymorphism; SVM: Support vector machines

\section{Acknowledgements}

We wish to thank Tomasz Stokowy for invaluable help in PTC dataset generation.

\section{Funding}

This research was financed by Polish National Centre for Research and Development within the programme PREVENTION AND TREATMENT OF CIVILIZATION DISEASES - STRATEGMEN III grant No.

STRATEGMED3/304586/5/NCBR/2017. The experiments were performed using the infrastructure supported by POIG.02.03.01-24-099/13 grant: "GeCONil-Upper Silesian Center for Computational Science and Engineering". The work was carried out in part within the statutory research project of the Institute of Informatics (RAU2). The funding bodies had no role in the design and the conclusions of the presented study.

\section{Availability of data and materials}

The datasets investigated in the study are publicly available (see the references from Table 1). Detailed information can be found in the project webpage (http://www.adaa.polsl.pl/software.html).

\section{Authors' contributions}

All the authors designed and implemented the algorithm, carried out the experiments and analyzed their results, prepared and approved the final manuscript.

\section{Competing interests}

The authors declare that they have no competing interests.

\section{Consent for publication}

Not applicable.

Ethics approval and consent to participate Not applicable.

\section{Publisher's Note}

Springer Nature remains neutral with regard to jurisdictional claims in published maps and institutional affiliations.

\section{Author details}

${ }^{1}$ Institute of Informatics, Silesian Univ. of Technology, Akademicka 16, 44-100 Gliwice, Poland. ${ }^{2}$ Institute of Innovative Technologies, EMAG, Leopolda 31, 40-189 Katowice, Poland.

Received: 16 August 2016 Accepted: 18 May 2017

Published online: 30 May 2017

\section{References}

1. Kalbfleisch JD, Prentice RL, Vol. 360. The Statistical Analysis of Failure Time Data. Hoboken: John Wiley \& Sons; 2011.

2. Cox DR. Regression models and life-tables. J R Stat Soc Series B (Methodological). 1972;34(2):187-220.

3. Holmes G, Hall M, Frank E. Generating Rule Sets from Model Trees In: Foo N, editor. Advanced Topics in Artificial Intelligence. LNCS. Berlin Heidelberg: Springer; 1999. p. 1-12.

4. Janssen F, Fürnkranz J. Heuristic Rule-Based Regression via Dynamic Reduction to Classification In: Walsh T, editor. IJCAl-11. Menlo Park: AAAI Press; 2011. p. 1330-5.

5. Sikora M, Skowron A, Wróbel L. Rule quality measure-based induction of unordered sets of regression rules In: Ramsay A, Agre G, editors. Artificial Intelligence: Methodology, Systems, and Applications. LNCS. Berlin Heidelberg: Springer; 2012. p. 162-71.

6. Harrington DP, Fleming TR. A class of rank test procedures for censored survival data. Biometrika. 1982;69(3):553-66.

7. Michalski RS. Discovering classification rules using variable-valued logic system VL. In: IJCAl-73. San Francisco: William Kaufmann; 1973. p. 162-72.

8. Fürnkranz J. Separate-and-conquer rule learning. Artif Intell Rev. 1999;13(1):3-54.

9. Pattaraintakorn P, Cercone N. A foundation of rough sets theoretical and computational hybrid intelligent system for survival analysis. Comput Math Appl. 2008;56(7):1699-708.

10. Bazan JG, Osmólski A, Skowron A, Ślezak D, Szczuka MS, Wróblewski 」. Rough set approach to the survival analysis In: Alpigini JJ, Peters JF, Skowronek J, Zhong N, editors. Rough Sets and Current Trends in Computing. LNCS. Berlin Heidelberg: Springer; 2002. p. 522-9.

11. Sikora M, Wróbel L, Mielcarek M, Kawłak K. Application of rule induction to discover survival factors of patients after bone marrow transplantation. J Med Inform Technol. 2013;22:35-53.

12. Kronek LP, Reddy A. Logical analysis of survival data: prognostic survival models by detecting high-degree interactions in right-censored data. Bioinformatics. 2008;24(16):248-53.

13. Chikalov I, Lozin V, Lozina I, Moshkov M, Nguyen H, Skowron A, Zielosko B. Logical analysis of data: Theory, methodology and applications. In: Three Approaches to Data Analysis. Intelligent Systems Reference Library. Berlin Heidelberg: Springer; 2013. p. 147-92.

14. Crama Y, Hammer PL, Ibaraki T. Cause-effect relationships and partially defined boolean functions. Ann Oper Res. 1988;16(1):299-325.

15. Liu X, Minin V, Huang Y, Seligson DB, Horvath S. Statistical methods for analyzing tissue microarray data. J Biopharm Stat. 2004;14(3):671-85.

16. LeBlanc M, Crowley J. Relative risk trees for censored survival data. Biometrics. 1992;48(2):411-25.

17. Therneau TM, Grambsch PM, Fleming TR. Martingale-based residuals for survival models. Biometrika. 1990;77(1):147-60. 
18. Wróbel $Ł$. Tree-based induction of decision list from survival data. J Med Inform Technol. 2012;20:73-8.

19. Frank E, Witten $\mid H$. Generating accurate rule sets without global optimization. In: ICML-98. San Mateo: Morgan Kaufmann; 1998. p. 144-51.

20. Wróbel $Ł$, Sikora M. Censoring weighted separate-and-conquer rule induction from survival data. Method Inform Med. 2014;53(2):137-48.

21. An A, Cercone N. Rule quality measures for rule induction systems: description and evaluation. Comput Intell. 2001;17(3):409-24.

22. Janssen F, Fürnkranz J. On the quest for optimal rule learning heuristics. Mach Learn. 2010;78(3):343-79.

23. Sikora $M$, Wróbel $Ł$. Data-driven adaptive selection of rule quality measures for improving rule induction and filtration algorithms. Int J Gen Syst. 2013;42(6):594-613.

24. Segal MR. Regression trees for censored data. Biometrics. 1988;44(1):35-47.

25. LeBlanc M, Crowley J. Survival trees by goodness of split. J Amer Statist Assoc. 1993;88(422):457-67.

26. Bou-Hamad I, Larocque D, Ben-Ameur H. A review of survival trees. Stat Surv. 2011:5:44-71.

27. Faraggi D, Simon R. A neural network model for survival data. Stat Med. 1995; 14(1):73-82.

28. Biganzoli E, Boracchi P, Mariani L, Marubini E. Feed forward neural networks for the analysis of censored survival data: a partial logistic regression approach. Stat Med. 1998;17(10):1169-86.

29. Ripley RM, Harris AL, Tarassenko L. Non-linear survival analysis using neural networks. Stat Med. 2004;23(5):825-42.

30. Štajduhar I, Dalbelo-Bašić B, Bogunović N. Impact of censoring on learning bayesian networks in survival modelling. Artif Intell Med. 2009;47(3):199-217.

31. Štajduhar I, Dalbelo-Bašić B. Learning bayesian networks from survival data using weighting censored instances. J Biomed Inform. 2010;43(4): $613-22$.

32. Van Belle V, Pelckmans K, Van Huffel S, Suykens JA. Improved performance on high-dimensional survival data by application of Survival-SVM. Bioinformatics. 2011;27(1):87-94.

33. Binder $H$, Allignol A, Schumacher $M$, Beyersmann J. Boosting for high-dimensional time-to-event data with competing risks. Bioinformatics. 2009;25(7):890-6.

34. Ishwaran H, Kogalur UB, Blackstone EH, Lauer MS. Random survival forests. Ann Appl Statist. 2008;2(3):841-60.

35. Hothorn T, Lausen B, Benner A, Radespiel-Tröger M. Bagging survival trees. Stat Med. 2004;23(1):77-91.

36. Breiman L, Friedman JH, Olshen RA, Stone CJ. Classification and Regression Trees. Belmont: Wadsworth; 1984.

37. Radespiel-Troger M, Gefeller O, Rabenstein T, Hothorn T. Association between split selection instability and predictive error in survival trees. Method Inform Med. 2006;45(5):548-56.

38. Molinaro AM, Dudoit S, Van der Laan MJ. Tree-based multivariate regression and density estimation with right-censored data. J Multivar Anal. 2004;90(1):154-77.

39. Hothorn T, Hornik K, Zeileis A. Unbiased recursive partitioning: A conditional inference framework. J Comp Graph Stat. 2006;15(3):651-74.

40. Breiman L. Bagging predictors. Mach Learn. 1996;24(2):123-40.

41. Breiman L. Random forests. Mach Learn. 2001;45(1):5-32.

42. Benner A. Application of "aggregated classifiers" in survival time studies In: Compstat. Heidelberg: Springer; 2002. p. 171-6.

43. Hothorn T, Buehlmann P, Dudoit S, Molinaro A, Laan MVD. Survival ensembles. Biostatistics. 2006;7(3):355-73.

44. Zhou L, Xu Q, Wang $\mathrm{H}$. Rotation survival forest for right censored data. PeerJ. 2015;3:1009.

45. Zhou Y, McArdle JJ. Rationale and applications of survival tree and survival ensemble methods. Psychometrika. 2015;80(3):811-33.

46. Schumacher M, Holländer N, Schwarzer G, Sauerbrei W. Prognostic Factor Studies In: Crowley J, Ankerst DP, editors. Prognostic Factor Studies. 2nd edn. Boca Raton: Chapman \& Hall/CRC; 2006. p. 289-333.

47. Kalwak K, Porwolik J, Mielcarek M, Gorczynska E, Owoc-Lempach J, Ussowicz M, Dyla A, Musial J, Pazdzior D, Turkiewicz D, Chybicka A. Higher $\mathrm{cd} 34(+)$ and $\mathrm{cd} 3(+)$ cell doses in the graft promote long-term survival, and have no impact on the incidence of severe acute or chronic graft-versus-host disease after in vivo t cell-depleted unrelated donor hematopoietic stem cell transplantation in children. Biol Blood Marrow Transplant. 2010;16(10):1388-401.
48. Beer DG, Kardia SL, Huang CC, Giordano TJ, Levin AM, Misek DE, Lin L, Chen G, Gharib TG, Thomas DG, et al. Gene-expression profiles predict survival of patients with lung adenocarcinoma. Nat Med. 2002;8(8): 816-24.

49. Network CGAR, et al. Integrated genomic characterization of papillary thyroid carcinoma. Cell. 2014;159(3):676-90.

50. Kaplan EL, Meier P. Nonparametric estimation from incomplete observations. J Am Stat Assoc. 1958;53(282):457-81.

51. Little RJ, Rubin DB. Statistical Analysis with Missing Data. vol. 539. New York: Wiley; 1987

52. Wohlrab L, Fürnkranz J. A review and comparison of strategies for handling missing values in separate-and-conquer rule learning. J Intell Inf Sys. 2011;36(1):73-98.

53. Schumacher M, Bastert G, Bojar H, Hübner K, Olschewski M, Sauerbrei W, Schmoor C, Beyerle C, Neumann R, Rauschecker H, et al. Randomized $2 \times 2$ trial evaluating hormonal treatment and the duration of chemotherapy in node-positive breast cancer patients. german breast cancer study group. J Clin Oncol. 1994;12(10):2086.

54. cBioPortal of Cancer Genomics. Papillary Thyroid Carcinoma. http://www. cbioportal.org/study?id=thca_tcga_pub. Accessed 18 May 2017.

55. Therneau T, Atkinson B, Ripley B. Rpart: Recursive Partitioning. 2013. R package version 4.1-1, http://CRAN.R-project.org/package=rpart. Accessed 18 May 2017.

56. GrafE, Schmoor C, Sauerbrei W, Schumacher M. Assessment and comparison of prognostic classification schemes for survival data. Stat Med. 1999;18(17-18):2529-45.

57. Schumacher M, GrafE, Gerds T, et al. How to assess prognostic models for survival data: a case study in oncology. Method Inform Med. 2003;42(5):564-71.

58. Demšar J. Statistical comparisons of classifiers over multiple data sets J Mach Learn Res. 2006;7:1-30.

59. Garcia S, Herrera F. An extension on "statistical comparisons of classifiers over multiple data sets" for all pairwise comparisons. J Mach Learn Res. 2008;9:2677-694

60. García S, Fernández A, Luengo J, Herrera F. Advanced nonparametric tests for multiple comparisons in the design of experiments in computational intelligence and data mining: Experimental analysis of power. Inform Sci. 2010;180(10):2044-64.

61. Benjamini $Y$, Hochberg Y. Controlling the false discovery rate: A practical and powerful approach to multiple testing. J R Stat Soc Series B (Methodological). 1995;57(1):289-300.

62. Fukushima T, Suzuki S, Mashiko M, Ohtake T, Endo Y, Takebayashi Y, Sekikawa K, Hagiwara K, Takenoshita S. BRAF mutations in papillary carcinomas of the thyroid. Oncogene. 2003;22(41):6455-7.

63. Hosmer DW, Lemeshow S, May S. Applied survival analysis: regression modeling of time to event data. New York: Wiley; 2008.

64. Loprinzi CL, Laurie JA, Wieand HS, Krook JE, Novotny PJ, Kugler JW Bartel J, Law M, Bateman M, Klatt NE. Prospective evaluation of prognostic variables from patient-completed questionnaires. north central cancer treatment group. J Clin Oncol. 1994;12(3):601-7.

65. Pintilie M. Competing Risks: a Practical Perspective. vol. 58. New York: John Wiley \& Sons; 2006.

66. Lange N, Ryan L, Billard L, Brillinger D, Conquest L, Greenhouse J. Case Studies in Biometry. Wiley series in probability and mathematical statistics: Applied probability and statistics. New York: Wiley; 1994

67. Andersen PK, Borgan O, Gill RD, Keiding N. Statistical Models Based on Counting Processes. Berlin Heidelberg: Springer; 2012.

68. Kyle RA. "Benign" monoclonal gammopathy-after 20 to 35 years of follow-up. Mayo Clin Proc. 1993;68(1):26-36.

69. Fleming TR, Harrington DP. Counting Processes and Survival Analysis. vol. 169. New York: John Wiley \& Sons; 2011.

70. Klein JP, Moeschberger ML. Survival Analysis: Techniques for Censored and Truncated Data. Berlin Heidelberg: Springer; 2005.

71. Rosenman RH, Friedman M, Jenkins CD, Straus R, Wurm M, Kositchek R. Clinically unrecognized myocardial infarction in the western collaborative group study. Am J Cardiol. 1967;19(6):776-82.

72. Abnet CC, Lai B, Qiao YL, Vogt S, Luo XM, Taylor PR, Dong ZW, Mark SD, Dawsey SM. Zinc concentration in esophageal biopsy specimens measured by $x$-ray fluorescence and esophageal cancer risk. J Natl Cancer Inst. 2005;97(4):301-6. 